\title{
Visual outcomes in Patients with Diabetic Macular Oedema undergoing Cataract Surgery with or without Intravitreal Bevacizumab
}

\author{
Mazhar Hassan Hassan, Aziz Ur Rehman, Umair Qidwai, Rashid Alvi, Nasir Bhatti, Ashraf \\ Dawood \\ Al-Ibrahim Eye Hospital/Isra Postgraduate Institute of Ophthalmology, Karachi, Pakistan
}

\begin{abstract}
Aims: To evaluate the efficacy of a single intravitreal bevacizumab injection after cataract surgery for the management of postoperative decrease in vision in patients with diabetic macular oedema.

Methods: In this randomised controlled open-label parallel group study of 60 patients with diabetic macular oedema and lens opacity (grade $\geq 3$ ), 30 eyes received a single intravitreal bevacizumab injection after cataract surgery, and 30 control eyes did not receive bevacizumab. The primary endpoint was change in best-corrected visual acuity 6 weeks after operation compared with that at baseline using the Snellen visual acuity chart.

Results: Postoperative visual acuity was significantly different between the group receiving bevacizumab and the control group $(p<0.005)$. All patients in the bevacizumab group had postoperative visual acuities above 6/18 with 27 patients having visual acuities of 6/12 or better compared with 6 patients in the control group. None of the patients in the bevacizumab group had visual acuities less than 6/18 compared with 11 patients in the control group.

Conclusion: Intravitreal bevacizumab after cataract surgery appears to be beneficial for preventing postoperative visual loss in eyes with diabetic retinopathy by reducing the risk of macular thickening.
\end{abstract}

Key words: Bevacizumab, Macular edema, Visual acuity

Asian J Ophthalmol. 2011;12:135-9.

\section{Introduction}

Visual outcomes following cataract surgery in patients with diabetes depends primarily on the presence of macular oedema. ${ }^{1,2}$ Patients with diabetes have developed severe maculopathy following cataract surgery. ${ }^{3}$ As it is important to be able to predict the long-term visual effects before cataract surgery is performed, surgeons need to have a better understanding of the natural course of diabetic macular oedema (DM0) as well as diabetic retinopathy (DR) after cataract surgery.

DM0 has been shown to worsen after cataract surgery, ${ }^{4-6}$ although controversy remains as to the incidence of this worsening. ${ }^{7,8}$

Correspondence: Dr Mazhar Hassan Hassan, Al-lbrahim Eye Hospital/ Isra Postgraduate Institute of Ophthalmology, Old Thana, Malir, Karachi, Pakistan.

Tel: (92 307) 222 4048;

E-mail: drmazharulhassan@yahoo.com
It has also been suggested that macular oedema tends to worsen in eyes affected by DR at the time of cataract surgery. ${ }^{9}$ Distinguishing transient oedema from substantial progression of maculopathy is important for the timing of treatment of macular oedema, which includes laser photocoagulation, ${ }^{10}$ vitrectomy, ${ }_{1}^{11}$ and triamcinolone injection. ${ }^{12-14}$ Until recently, there have been no quantitative studies to examine the progression of DM0 after cataract surgery. A recent study by Kim et al showed a short-term increase in macular thickness after cataract surgery. ${ }^{15}$ It has been suggested that $22 \%$ of patients with diabetes develop increases in central retinal thickness after uncomplicated phacoemulsification. The presence of clinically significant macular oedema is a strong risk factor for subsequent macular thickening after surgery. ${ }^{15}$ Even if the visual prognosis is improved by cataract surgery, macula oedema remains a major risk factor for postoperative visual disturbance in patients with diabetes. 
Treatment to reduce the risk of postoperative macular thickening may improve visual outcomes in patients with diabetes. Laser photocoagulation remains the standard approach for treating DM0. ${ }^{16}$ However, it is sometimes difficult to obtain sufficient efficacy of laser treatment in patients with dense cataract. Trials of intravitreal or sub-Tenon triamcinolone acetonide ${ }^{17}$ and pars plana vitrectomy ${ }^{11}$ have been conducted, but no widely accepted technique has yet been established.

Vascular endothelial growth factor (VEGF) is considered to have a key role in the progression of abnormal angiogenesis, including DMO.$^{18}$ Hypoxia induces VEGF gene transcription, and elevated levels of VEGF have been found in ocular fluid of patients with DMO ${ }^{19}$ Bevacizumab is a humanised monoclonal antibody that inhibits all isoforms of VEGF. ${ }^{20}$ It has been reported that intravitreal injection of bevacizumab yields promising results in various neovascular eye diseases, including age-related macular degeneration, ${ }^{21}$ central retinal vein occlusion, ${ }^{22}$ and DM0 $0 .{ }^{23}$ Lanzagorta-Aresti et al have shown improvement in vision and decrease in retinal thickening in patients receiving bevacizumab compared with controls. ${ }^{24}$

This study was performed to evaluate the efficacy of a single intravitreal bevacizumab injection after cataract surgery for the management of postoperative decrease in vision in patients with DMO.

\section{Methods \\ Design}

This trial was a randomized controlled open-label parallel group study. Patients were recruited from the Al-Ibrahim Eye Hospital, Karachi, Pakistan, between July 2008 and July 2009. The trial was conducted according to the principles of the Declaration of Helsinki. Approval was obtained from the Ethics Committee at the Al-Ibrahim Eye Hospital. Each patient provided signed informed consent before study entry.

\section{Patients}

Adult patients (age, 20 years or older) with type 1 or type 2 diabetes mellitus were eligible for enrolment. All patients underwent complete ophthalmic examination, including best-corrected visual acuity (BCVA), slit-lamp biomicroscopy, fundoscopy, applanation tonometry, and fluorescein angiography before recruitment. Inclusion criteria were DMO occurring 3 to 18 months prior to study entry, significant lens opacity (grade $\geq 3$ for any type of cataract [cortical, nuclear, or posterior subcapsular]) by the Lens Opacities Classification System III, ${ }^{25}$ macular oedema involving the fovea, and $B C V A \leq 20 / 40$.

Exclusion criteria were history of ocular surgery and inflammation, presence of other ocular diseases, intraoperative complications such as posterior capsule rupture and severe iris damage, proliferative diabetic retinopathy, photocoagulation of the treated eye within the previous 12 months, and no previous intravitreal injection, including inhibitors or steroid.

\section{Randomisation and Masking}

Systematic randomised sampling was done by allocating alternate eyes into the control group or bevacizumab group. Neither the patients nor the investigators were masked, but the technicians who tested visual acuity, optometrists, and statistical analysers were masked to the treatment assignment.

\section{Treatment}

Bevacizumab was injected into the vitreous cavity immediately after phacoemulsification and intraocular lens (IOL) implantation, and progression of DMO was assessed by clinical examination and visual acuity testing.

The operative technique included complete continuous curvilinear capsulorrhexis and phacoemulsification through a 3.5$\mathrm{mm}$ corneoscleral incision, with intracapsular implantation of a foldable acrylic IOL, followed by a single intravitreal injection of bevacizumab. Bevacizumab was prepared by the institutional pharmacy as sterile filled and packed tuberculin syringes containing $0.05 \mathrm{~mL}(1.25 \mathrm{mg})$ bevacizumab, which was injected intravitreally using a 30-G needle. Postoperatively, all patients received similar routine medication, including topical application of diclofenac sodium (0.1\%) 3 times daily, moxifloxacin $0.3 \% 5$ times daily, and prednisolone $0.1 \% 3$ times daily for 3 months after surgery. Eyes in the control group received no bevacizumab injections.

\section{Outcome Measures and Follow-up}

The primary endpoint was change in BCVA from baseline at 6 weeks. BCVA was assessed by Snellen visual acuity chart. Patients were evaluated at baseline and at 1, 3, and 6 weeks. BCVA, intraocular pressure (IOP), slit-lamp biomicroscopy, and indirect ophthalmological measurements were performed at each visit; fluorescein angiography was performed at baseline and at 6 weeks

\section{Statistical Analysis}

30 eyes in each group were required to achieve a power of $80 \%$ based on unpaired Student $t$ test with a 2-sided significance level of 0.05. Values are expressed as mean (SD). The significance of the differences between the intervention group and the control group data was assessed by unpaired Student $t$ test, and that between the pretreatment and post-treatment data within the same group was assessed by paired Student $t$ test. All statistical analyses were performed by using the Statistical Package for the Social Sciences 
version 17.0 (SPSS, Inc, Chicago, USA). A p value of $<0.05$ was considered to be statistically significant.

\section{Results}

Sixty eyes of 60 patients were examined, all of whom fulfilled the inclusion criteria. Thirty two patients (53.3\%) were men and 28 $(46.7 \%)$ were women. The mean age was 58.3 years (SD, 7.35 years; range, $45-83$ years).

There were no significant differences between the 2 groups in age, sex, and duration of diabetes. There were no significant differences in BCVA at baseline between the groups.

Postoperative visual acuity was significantly different between the group receiving bevacizumab and the control group $(p<0.005)$. All patients in the bevacizumab group had postoperative visual acuities above 6/18 with 27 patients having visual acuities of $6 / 12$ or better compared with 6 patients in the control group. None of the patients in the bevacizumab group had visual acuities less than 6/18 compared with 11 patients in the control group. Comparisons of the pre- and postoperative visual acuities in both groups are shown in Figures 1 and 2.

Only 4 eyes developed subconjunctival haemorrhage as a complication of bevacizumab injection. No other complications were noted.

\section{Discussion}

Several studies have shown that intravitreal injection of bevacizumab is useful in the management of DM0. ${ }^{23,26}$ Similarly, studies have shown that cataract surgery leads to noticeable thickening of the retina, implying that operative invasion enhances retinal vascular permeability because localised retinal oedema is caused by focal leakage from microaneurysms and dilated capillary segments. ${ }^{27}$ Many inflammatory mediators such as VEGF cause breakdown of the blood-retinal barrier. ${ }^{28,29}$ Patel et al reported that VEGF levels in aqueous obtained from patients with diabetes 1 day after surgery were approximately 10 -fold higher than those from control patients. ${ }^{30}$ Bevacizumab inhibits VEGF, which is a potent

Figure 1. Pre- and postoperative visual acuity distribution in the bevacizumab group $(n=30)$.

Abbreviation: $\mathrm{CF}=$ counting fingers.

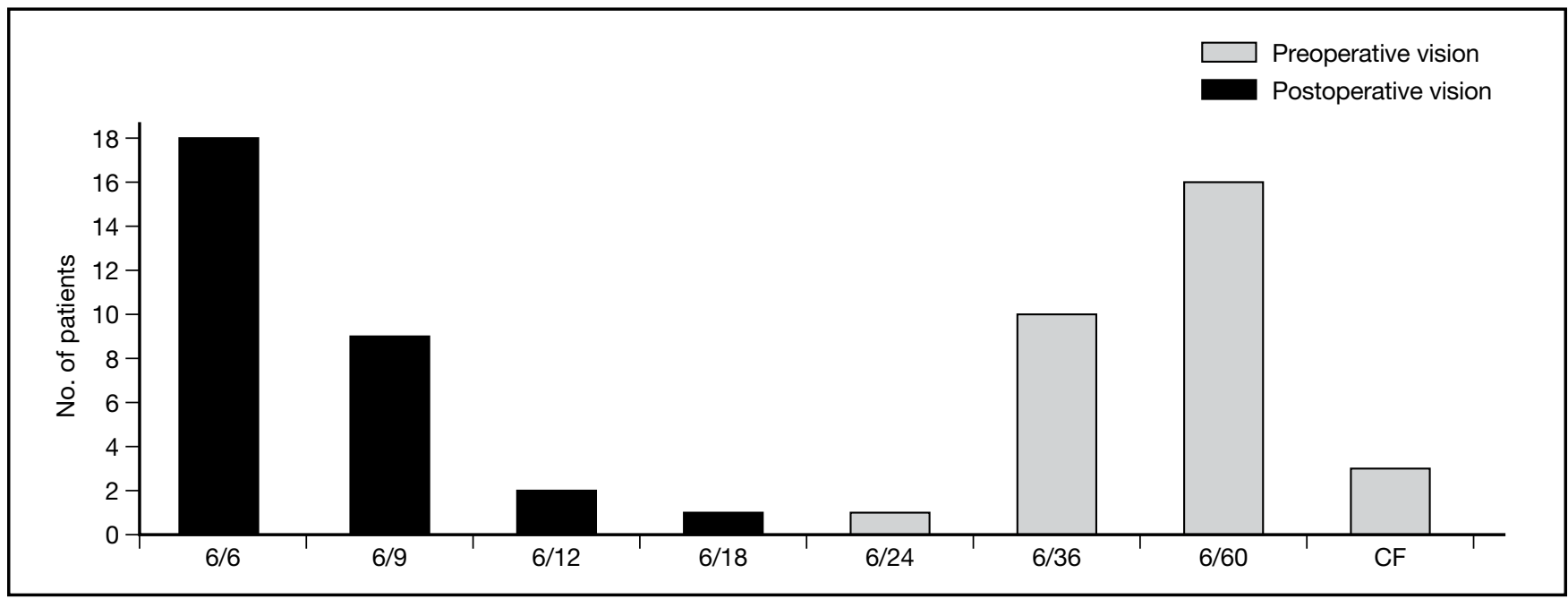

Figure 2. Pre- and postoperative visual acuity distribution in the control group $(n=30)$.

Abbreviation: $\mathrm{CF}=$ counting fingers; $\mathrm{HM}=$ hand movements.

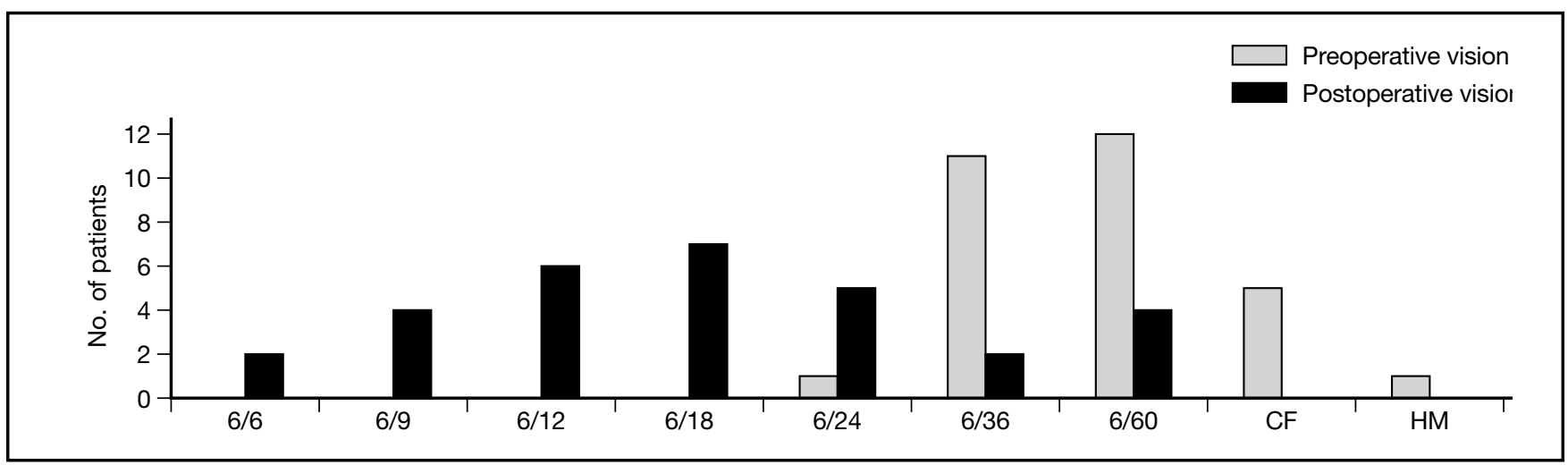


permeability factor implicated in cystoid macular oedema (CM0). ${ }^{29}$ Thus, this study hypothesised that anti-VEGF therapy would help to prevent the development of macular oedema after cataract surgery in patients with diabetes. A similar study by LanzagortaAresti et al showed improved vision and decreased retinal thickening in the bevacizumab group compared with the control group. ${ }^{24}$

Recently, studies have shown the clinical effect of intravitreal bevacizumab for pseudophakic CM0. ${ }^{31-33}$ Mason et al reported on 2 patients with persistent $\mathrm{CMO}$ who had been effectively treated with bevacizumab, and both eyes showed noticeable improvement in visual acuity. ${ }^{31}$ Similarly, Arevalo et al's study of 25 patients with pseudophakic CMO undergoing cataract surgery found a significant improvement in vision and decreased macular thickness. ${ }^{32}$ However, Spitzer et al reported that, although $81 \%$ of patients had significant improvement in central retinal thickness, visual outcome was not evidently improved. ${ }^{33}$ Therefore, the clinical effectiveness of intravitreal bevacizumab for pseudophakic CMO remains controversial. These case series excluded patients with diabetes, and there were several weeks between the day of cataract surgery and intravitreal bevacizumab therapy. In contrast, in this study, bevacizumab was injected into the vitreous cavity on the same day as the cataract surgery, immediately after IOL implantation. These data suggest that immediate intravitreal injection of bevacizumab is effective for improving vision after cataract surgery in patients with DMO.

The natural course of macular oedema after cataract surgery can be self-limiting in some patients with diabetes. ${ }^{34}$ The increased retinal thickness in the first month tended to show a decrease by the third month, which is consistent with the results of intravitreal triamcinolone for DM0 ${ }^{35,36}$ Among the new treatments such as corticosteroids and anti-VEGF agents clinically available for DME, laser photocoagulation remains the standard therapy and the only treatment with proven efficacy in a large clinical trial. ${ }^{10,16}$ Even though this study has shown bevacizumab to be effective, laser photocoagulation should be considered for the treatment of DMO for most patients, with the exception of those with dense cataract.

The dose of bevacizumab evaluated in this study was 1.25 $\mathrm{mg}$, which is that used most commonly in clinical practice. ${ }^{21-26}$ However, as no dose-ranging studies have been done, the ideal intravitreal concentration remains to be determined. Recurrence of CMO is possible and may require additional multiple injections of bevacizumab. Although there was no recurrent CMO in this study, the long-term efficacy is currently unknown.

One of the limitations of this study is the use of an observation group as a control, rather than a placebo injection, thereby making it impossible to ensure that the patients and investigators were masked to treatment. However, this limitation was mitigated by ensuring that the technicians who performed the visual acuity tests were masked. Other limitations of this study are that it was performed at a single centre, and that it involved individuals of only one race, factors that limit its generalisability to the general population. Another important limitation of this study was the unavailability of optical coherence tomography at the hospital, meaning that the retinal thickness of patients in this study could not be measured.

Although further investigation with a longer follow-up duration and a larger series of patients is needed, anti-VEGF therapy may be a potent tool for the treatment of DM0 after cataract surgery. Bevacizumab contributed to the significant improvement in visual acuity 6 weeks after cataract surgery in this study. Therefore, it is possible that intravitreal bevacizumab has the potential not only to prevent the progression of DM0 after cataract surgery, but also to improve its severity. Several reports have indicated that intravitreal or posterior sub-Tenon triamcinolone acetonide or corticosteroid treatment is also effective for reducing macula thickness in DM0. ${ }^{37-40}$ Shimura et al compared the effect of intravitreal injection of bevacizumab with triamcinolone acetonide for $\mathrm{DMO}$, and found that the triamcinolone treatment yielded better results in terms of macular thickness reduction and improvement in visual acuity. ${ }^{41}$ However, these authors also found a significant postoperative increase in IOP in the triamcinolone injected eyes, whereas the bevacizumab-treated eyes showed no significant change. ${ }^{41}$ As one of the most important side effects of triamcinolone treatment is elevation of IOP, bevacizumab may be beneficial for patients who are known steroid responders and who are unresponsive to nonsteroidal anti-inflammatory drugs. In this small case series, there was no significant increase in IOP postoperatively, and no eyes had infection or other serious ocular complications. However, a larger number of patients is needed to verify the safety of bevacizumab treatment.

In summary, intravitreal bevacizumab immediately after phacoemulsification prevents exacerbation of the macular oedema seen in many patients with diabetes undergoing cataract surgery.

\section{References}

1. Joussen AM, Smyth N, Niessen C. Pathophysiology of diabetic macular edema. Dev Ophthalmol. 2007;39:1-12.

2. Zaczek A, Olivestedt G, Zetterstrom C. Visual outcome after phacoemulsification and IOL implantation in diabetic patients. $\mathrm{Br} \mathrm{J}$ Ophthalmol. 1999;83:1036-41.

3. Benson WE, Brown GC, Tasman W, McNamara JA, Vander JF. Extracapsular cataract extraction with placement of a posterior chamber lens in patients with diabetic retinopathy. Ophthalmology. 1993;100:730-8.

4. Dowler JG, Sehmi KS, Hykin PG, Hamilton AM. The natural history of macular edema cataract surgery in diabetes. Ophthalmology. 1999;106:663-8. 
5. Dowler JG, Hykin PG, Hamilton AM. Phacoemulsification versus extracapsular cataract extraction in patients with diabetes. Ophthalmology. 2000;107:457-62.

6. Funatsu $\mathrm{H}$, Yamashita $\mathrm{H}$, Noma $\mathrm{H}$, Shimizu E, Mimura T, Hori S. Prediction of macular edema exacerbation after phacoemulsification in patients with nonproliferative diabetic retinopathy. J Cataract Refract Surg. 2002;28:1355-63.

7. Early Treatment Diabetic Retinopathy Study Research Group. Results after lens extraction in patients with diabetic retinopathy: Early Treatment Diabetic Retinopathy Study report number 25. Arch Ophthalmol. 1999;117:1600-6.

8. Squirrell D, Bhola R, Bush J, Winder S, Talbot JF. A prospective, case controlled study of the natural history of diabetic retinopathy and maculopathy after uncomplicated phacoemulsification cataract surgery in patients with type 2 diabetes. $\mathrm{Br} \mathrm{J}$ Ophthalmol. 2002;86:565-71.

9. Henricsson M, Heijl A, Janzon L. Diabetic retinopathy before and after cataract surgery. Br J Ophthalmol. 1996;80:789-93.

10. Early Treatment Diabetic Retinopathy Study Research Group. Photocoagulation for diabetic macular edema: Early Treatment Diabetic Retinopathy Study report number 1. Arch Ophthalmol. 1985;103:1796-806.

11. Tachi N, Ogino N. Vitrectomy for diffuse macular edema in cases of diabetic retinopathy. Am J Ophthalmol. 1996;122:258-60.

12. Jonas JB, Sofker A. Intraocular injection of crystalline cortisone as adjunctive treatment of diabetic macular edema. Am J Ophthalmol. 2001;132:425-7.

13. Martidis A, Duker JS, Greenberg PB, et al. Intravitreal triamcinolone for refractory diabetic macular edema. Ophthalmology. 2002;109:920-7.

14. Jonas JB, Kreissig I, Sofker A, Degenring RF. Intravitreal injection of triamcinolone for diffuse diabetic macular edema. Arch Ophthalmol. 2003;121:57-61.

15. Kim SJ, Equi R, Bressler NM. Analysis of macular edema after cataract surgery in patients with diabetes using optical coherence tomography. Ophthalmology. 2007;114:881-9.

16. Writing Committee for the Diabetic Retinopathy Clinical Research Network. Comparison of the modified Early Treatment Diabetic Retinopathy Study and mild macular grid laser photocoagulation strategies for diabetic macular edema. Arch Ophthalmol. 2007; 125:469-80.

17. Conway MD, Canakis C, Livir-Rallatos C, Peyman GA. Intravitreal triamcinolone acetonide for refractory chronic pseudophakic cystoid macular edema. J Cataract Refract Surg. 2003;29:27-33

18. Ferrara N. Vascular endothelial growth factor: basic science and clinical progress. Endocr Rev. 2004;25:581-611.

19. Funatsu H, Yamashita H, Nakamura S, Mimura T, Nakamura S, Hori $\mathrm{S}$. Vitreous levels of pigment epithelium-derived factor and vascular endothelial growth factor are related to diabetic macula edema. Ophthalmology. 2006;113:294-301.

20. Ferrara N, Hillan KJ, Gerber HP, Novotny W. Discovery and development of bevacizumab, an anti-VEGF antibody for treating cancer. Nat Rev Drug Discov. 2004;3:391-400.

21. Avery RL, Pieramici DJ, Rabena MD, Castellarin AA, Nasir MA, Giust MJ. Intravitreal bevacizumab (Avastin) for neovascular age-related macular degeneration. Ophthalmology. 2006;113:363-72.

22. Iturralde D, Spaide RF, Meyerle CB, et al. Intravitreal bevacizumab (Avastin) treatment of macular edema in central retinal vein occlusion: a short-term study. Retina. 2006;26:279-84.

23. Arevalo JF, Fromow-Guerra J, Quiroz-Mercado H, et al, PanAmerican Collaborative Retina Study Group. Primary intravitreal bevacizumab (Avastin) for diabetic macular edema: results from the Pan-American Collaborative Retina Study Group at 6-month follow-up. Ophthalmology. 2007;114:743-50.
24. Lanzagorta-Aresti A, Palacios-Pozo E, Menezo R, Jose L, NaveaTejerina A. Prevention of vision loss after cataract surgery in diabetic macular edema with intravitreal bevacizumab: a pilot study. Retina. 2009:29:530-5.

25. Chylack LT Jr, Wolfe JK, Singer DM, et al, Longitudinal Study of Cataract Study Group. The Lens Opacities Classification System III. Arch Ophthalmol. 1993;111:831-6.

26. Diabetic Retinopathy Clinical Research Network. A phase II randomized clinical trial of intravitreal bevacizumab for diabetic macular edema. Ophthalmology. 2007;114:1860-7.

27. Cunha-Vaz JG. Studies on the pathophysiology of diabetic retinopathy: the blood-retinal barrier in diabetes. Diabetes. 1983; 32(Suppl):S20-7.

28. Stern AL, Taylor DM, Dalburg LA, Cosentino RT. Pseudophakic cystoid maculopathy: a study of 50 cases. Ophthalmology. 1981; 88:942-6.

29. Qaum T, Xu Q, Joussen AM, et al. VEGF-initiated bloodretinal barrier breakdown in early diabetes. Invest Ophthalmol Vis Sci. 2001;42:2408-13.

30. Patel JI, Hykin PG, Cree IA. Diabetic cataract removal: postoperative progression of maculopathy - growth factor and clinical analysis. Br J Ophthalmol. 2006;90:697-701.

31. Mason JO III, Albert MA Jr, Vail R. Intravitreal bevacizumab (Avastin) for refractory pseudophakic cystoid macular edema. Retina. 2006;26:356-7.

32. Arevalo JF, Garcia-Amaris RA, Roca JA, et al, Pan-American Collaborative Retina Study Group. Primary intravitreal bevacizumab for the management of pseudophakic cystoid macular edema: pilot study of the Pan-American Collaborative Retina Study Group. J Cataract Refract Surg. 2007;33:2098-105.

33. Spitzer MS, Ziemssen F, Yoeruek E, Petermeier K, Aisenbrey S, Szurman P. Efficacy of intravitreal bevacizumab in treating postoperative pseudophakic cystoid macular edema. J Cataract Refract Surg. 2008;34:70-5.

34. Kim SJ, Equi R, Bressler NM. Analysis of macular edema after cataract surgery in patients with diabetes using optical coherence tomography. Ophthalmology. 2007;114:881-9.

35. Diabetic Retinopathy Clinical Research Network. A randomized trial comparing intravitreal triamcinolone acetonide and focal/grid photocoagulation for diabetic macular edema. Ophthalmology. 2008;115:1447-59.

36. Diabetic Retinopathy Clinical Research Network. Randomized trial of peribulbar triamcinolone acetonide with and without focal photocoagulation for mild diabetic macular edema: a pilot study. Ophthalmology. 2007;114:1190-6.

37. Jonas JB, Kreissig I, Söfker A, Degenring RF. Intravitreal injection of triamcinolone for diffuse diabetic macular edema. Arch Ophthalmol. 2003;121:57-61.

38. Sutter FK, Simpson JM, Gillies MC. Intravitreal triamcinolone for diabetic macular edema that persists after laser treatment: threemonth efficacy and safety results of a prospective, randomized, double-masked, placebo-controlled clinical trial. Ophthalmology. 2004;111:2044-9

39. Massin P, Audren F, Haouchine B, et al. Intravitreal triamcinolone acetonide for diabetic diffuse macular edema: preliminary results of a prospective controlled trial. Ophthalmology. 2004;111: 218-24.

40. Martidis A, Duker JS, Greenberg PB, et al. Intravitreal triamcinolone for refractory diabetic macular edema. Ophthalmology. 2002;109: 920-7.

41. Shimura M, Nakazawa T, Yasuda K, et al. Comparative therapy evaluation of intravitreal bevacizumab and triamcinolone acetonide on persistent diffuse diabetic macular edema. Am J Ophthalmol. 2008;145:854-61. 